\title{
Fulvio DELLE DONNE, La porta del sapere : cultura alla corte di Federico II di Svevia
}

Jean-Paul Boyer

\section{(2) OpenEdition}

\section{Journals}

Édition électronique

URL : https://journals.openedition.org/ccm/8017

DOI : $10.4000 / \mathrm{ccm} .8017$

ISSN : 2119-1026

\section{Éditeur}

Centre d'études supérieures de civilisation médiévale/Université de Poitiers

\section{Édition imprimée}

Date de publication : 1 septembre 2021

Pagination : 259-262

ISBN : 978-2-490783-10-6

ISSN : 0007-9731

\section{Référence électronique}

Jean-Paul Boyer, "Fulvio delle donne, La porta del sapere : cultura alla corte di Federico I/ di Svevia », Cahiers de civilisation médiévale [En ligne], 255 | 2021, mis en ligne le 01 septembre 2021, consulté le 05 février 2022. URL : http://journals.openedition.org/ccm/8017; DOI : https://doi.org/10.4000/ccm. 8017

\section{(c) (i) (9)}

La revue Cahiers de civilisation médiévale est mise à disposition selon les termes de la Licence Creative Commons Attribution - Pas d'Utilisation Commerciale - Pas de Modification 4.0 International. 
Fulvio Delle Donne, La porta del sapere : cultura alla corte di Federico II di Svevia, Rome, Carocci Editore (Frecce, 271), 2019.

Fulvio Delle Donne se fixe un objet aussi difficile qu'opportun en rouvrant le dossier sur Frédéric II de Souabe (1194-1250), l'illustre empereur et roi de Sicile (soit du Mezzogiorno). Sa tâche se fait encore plus ardue, comme utile, sous l'angle qu'il privilégie, annoncé par le titre de l'ouvrage, savoir la culture du monarque et de sa cour sous le rapport du politique. Ainsi l'a. soutient-il une double gageure.

Le défi le plus direct vient d'une production dorénavant diluvienne sur Frédéric II et son régime. D'amples et récentes vues d'ensemble, pour comble, la couronnent. Ne citons que les travaux de Wolfgang Stürner (Federico II e l'apogeo dell'impero, Andrea Antonion Verardi [éd.] et Ortensio Zecchino [préf.], Rome, Salerno Editrice [Biblioteca storica], 2009) et, surtout, l'Enciclopedia fridericiana (Massimo BrAY, et al. [dir.], Rome, Treccani, 2005-2008, 3 vol.). En trois volumes et environ 600 articles, cette authentique somme s'étend de Frédéric II au contexte le plus large de son règne. Pour toutes les questions abordées, elle offre les contributions des meilleurs spécialistes, qui paraissent donc définitives. Or, le bilan historiographique ne décourage pas que la quête de données qui eussent échappé à l'observation. Il induit une conséquence plus pernicieuse. Tant d'écrits convergent, en moyenne, pour imposer une quasi-vulgate sur les thèmes que reprend F. Delle Donne. Les convictions partagées portent principalement, en effet, sur la psychologie de l'empereur, sur son relief intellectuel et idéologique, sur celui de son entourage et sur leur programme de gouvernement.

Un long assentiment a conforté la réputation de Frédéric II comme individu et souverain au-dessus de l'habituelle condition humaine. Une aura fabuleuse s'attacha à lui de son vivant, en particulier comme hérétique et Antéchrist. Les légendes se perpétuèrent après sa mort, entre l'attente espérée de son retour et sa malédiction. De celle-ci, Dante prolongea l'écho en plaçant l'empereur entre les damnés parmi les épicuriens (Enfer, X, 119). Sa renommée sulfureuse servit cependant le mieux son prestige des Lumières à nos jours, au premier chef depuis le $\mathrm{XVIII}^{\mathrm{e}} \mathrm{s}$. napolitain. Pietro Giannone en fit alors un champion de l'autonomie de l'État face à l'Église. De là, il parut au XIX $\mathrm{s}$. un précurseur de l'unité italienne (Aurelio Musı, « Il mito di Federico II nell'Ottocento italiano », Il Risorgimento, 66, 2019, p. 11-27, DOI : 10.3280/ RISO2019-001002). Ernst Kantorowicz élabora enfin sur ces bases, en radicalisant leur schématisme, son portrait de Frédéric II (Kaiser Friedrich der Zweite, Berlin, Küpper-Bondi, 1927). Il éleva son héros, dans une perspective téléologique, au rang du surhomme nietzschéen, capable de rompre avec l'éthique de son époque. Il en fit le précurseur de l'État moderne, voire totalitaire, comme valeur suprême. Cette célébration jusqu'à la logorrhée, tirant d'indices parfois ténus des certitudes, n'a cessé de fasciner. Historiens ou philosophes pensent toujours trouver chez Frédéric II un exemple d'esprit fort et, simultanément, un pionnier du despotisme étatique. Parmi eux, en bref, les épigones d'E. Kantorowicz donnent encore le ton. Ainsi nombre d'auteurs acceptent-ils sans sourciller le prédicat de «transformateur du monde » qui eût été reconnu à l'empereur au XIII" $s$. même (Thomas Curtis Van Cleve, The Emperor Frederick II of Hohenstaufen, Immutator Mundi, Oxford, Clarendon Press, 1972).

Le présent livre repart justement de cette qualification dans sa forme originelle : Stupor mundi et immutator 
mirabilis (« stupeur du monde et son modificateur étonnant »), mais pour la réduire à une juste taille. Due au seul Matthieu Paris, chroniqueur anglais éloigné du monde de Frédéric II, elle trahit le reproche d'avoir perturbé l'équilibre de la société plus que l'admiration pour un inventeur de génie. F. Delle Donne annonce ainsi son intention de s'affranchir d'interprétations aventurées. Il a, au reste, consacré un précédent essai à l'occultation du vrai Frédéric II derrière des fictions sans cesse renouvelées (Federico II : la condanna della memoria. Metamorfosi di un mito, Rome, Viella, 2012). Il a ainsi dégagé le terrain pour l'étape aujourd'hui franchie, celle d'une réalité historique du monarque et de son entourage.

Certes, quelques publications ont déjà visé à les proportionner à leur siècle. Tel fut le dessein de David Abulafia dans sa mémorable biographie de Frédéric II. Il le rétablit en « empereur médiéval », car « il fut en réalité un homme de son temps et non ce despote de la Renaissance, avant la lettre, que la tradition nous a transmis » (Frederick II, a Medieval Emperor, Londres, Penguin Press, 1988; trad. italienne : Federico II : un imperatore medievale, Gianluigi Mainardi [trad.], Turin, Einaudi [Einaudi Tascabili, saggi, 120], 1993, p. 364). F. Delle Donne salue les acquis de cette enquête anticonformiste. Il en déplore, néanmoins, un excès d'iconoclasme quelquefois gratuit. Comme il se déduit de son propre ouvrage, il objecte au fond à $\mathrm{D}$. Abulafia le retour à des conceptions un peu trop positivistes, qui estomperaient la composante intellectuelle du règne de l'empereur, avec sa dimension remarquable à défaut d'extraordinaire.

De fait, tout en s'écartant de spéculations hasardeuses d'E. Kantorowicz, F. Delle Donne revendique son héritage en ce qu'il fut novateur et demeure exemplaire. Entendons, à la base, qu'il promut une histoire totale qui embrassât philosophie, théologie, doctrines politiques, littérature ou approche littéraire des sources, art et architecture. Ces fondements répondirent à un dépassement résolu du positivisme. Ce tournant épistémologique enseigna l'importance majeure, pour l'intelligence des sociétés, de la communication et des représentations, jusqu'aux mythes. Tels sont bien, dans leurs grandes lignes, les principes et les fins du présent livre.

Il revient sur les sources à fort contenu idéologique dont l'étude avait fait l'originalité d'E. Kantorowicz. Il conforte ses conclusions ou les écarte par une révision méticuleuse du dossier envisagé par son insigne devancier. S'il se réfère encore à une bibliographie très à jour, le retour systématique aux racines du sujet donne un prix singulier à son ouvrage. Son souci d'exactitude s'étend au contexte. Il en va ainsi pour l'atmosphère apocalyptique ou surnaturelle qui put envelopper Frédéric II. Elle garde une place dans l'exposé; mais F. Delle Donne en détermine les manifestations contemporaines à l'empereur et les explique comme congrues à son temps.

La relecture du corpus envisagé requérait la double qualité de l'a. d'éminent spécialiste de la littérature médiévale et d'historien du Mezzogiorno. Son nouvel ouvrage se distingue, à son tour, comme très savant, voire technique. À cet égard, le choix de l'éditeur de refuser les notes pour mêler au texte les multiples références, placées entre parenthèses, se révèle peu pratique. Il impose d'abréger les renvois aux sources et à la bibliographie. Le procédé hache encore la lecture. Heureusement, F. Delle Donne s'exprime avec une clarté parfaite. Il rend accessible à des néophytes jusqu'à ses considérations les moins faciles, ainsi sur la stylistique latine. Il suit, en sus, une démarche transparente.

Dans un premier chapitre, il brosse « le contexte historique », en accord avec l'importance qu'il lui reconnaît. Il passe ensuite à la sphère proprement idéologique et culturelle. Le gros du travail consiste dans l'examen en quatre chapitres des principaux témoignages ou interrogations sur Frédéric II et son cercle. Il s'agit d'une analyse critique menée source par source, point par point. Sans doute le développement en est-il fractionné, outre quelques rares chevauchements. Le retour aux origines de nos connaissances, sur l'empereur et sur son milieu, impose ce procès inductif. Il n'empêche pas de dégager peu à peu des vues d'ensemble. Elles aboutissent à une présentation plus globale de la « culture officielle » dans un ultime chapitre assez nourri. Quoi qu'il en soit, la multiplicité des apports de l'ouvrage ne permet pas de les résumer tous; mais quelques-uns valent spécialement de se signaler.

Au départ, la synthèse chronologique dégage les enjeux politiques du règne de Frédéric II. Selon le plan souabe déjà imaginé par son grand-père, Frédéric Barberousse, une ferme administration du royaume de Sicile devait s'ajouter et contribuer aux efforts pour imposer une supériorité effective sur les terres d'Empire. La combinaison prenait en tenailles 
une papauté post-grégorienne, qui s'estimait concurremment détentrice de l'autorité suprême jusqu'au temporel. Une lutte inévitable avec le Saint-Siège en résultait. L'action culturelle déployée par Frédéric II et par son entourage porte, à divers degrés, la marque de ce complexe d'ambitions et de contraintes.

L'ars dictaminis, comme elle se cultivait auprès du roi-empereur, en demeure le meilleur témoin. Aussi F. Delle Donne en privilégie-t-il les restes pour accéder à la culture politique et aux projets de Frédéric II et de ses proches. Il s'agissait d'une rhétorique appliquée à la rédaction des lettres. Elle s'étendait à la correspondance réelle ou fictive des lettrés ; mais elle relevait avant tout de la chancellerie, où elle atteignait son sommet sous la longue direction du célèbre Pierre des Vignes. Cet art s'entait sur une tradition campanienne. Nonobstant, il s'épanouit dans le cadre d'un affrontement avec la curie pontificale, qui avait un long usage du dictamen pour fonder son ascendant moral. Une vraie guerre des deux diplomatiques se déchaîna. De fait, les textes concernés se destinaient à la lecture à haute voix.

Leur centralité dans l'expression politique est soulignée par la marginalité, à l'inverse, d'autres vecteurs en soutien de Frédéric II. F. Delle Donne rappelle ici le déclin du genre de la chronique. Il dissipe encore certaines illusions quant à la poésie aulique en langue vulgaire, soit une sorte de « sicilien illustre». Si elle marqua le grand départ de la poésie italienne, vernaculaire, elle demeura une production privée de quelques lettrés. Elle découlait néanmoins de leur belle maîtrise de l'écrit.

Il y avait bien une contiguïté avec la conquête de l'opinion en partant de la forme, quant aux lettres émises par la cour de Frédéric II. Elles déployaient un langage recherché, majestueux, volontiers obscur, imprimant aux phrases une cadence harmonieuse (un cursus). Leur tournure inculquait un sentiment de révérence. Elle convainquait d'une domination intellectuelle, voire spirituelle. Savantes, hermétiques, grandiloquentes, les lettres tant de la curie que de la monarchie sicilienne convenaient pour véhiculer la teneur de prophéties dont le public était avide. Manié davantage par le Saint-Siège, le joachimisme alimentait alors les craintes apocalyptiques qui s'attachaient à l'empereur souabe. Dans son camp, les prétendues vaticinations des sibylles insufflaient l'espoir d'un retour à l'âge d'or. Toutefois, selon une vocation du dictamen qui partait des origines, le modèle politico-littéraire adopté supportait principalement discours ou argumentations juridiques.

F. Delle Donne démonte cette habile association dans l'examen du préambule au Liber Augustalis (1231), la compilation de lois applicables au royaume de Sicile. Sa composante littéraire, voire théologique, assurait de l'autorité normative du roi et empereur. Le rôle du droit se manifestait autant dans la fondation du studium (groupe d'écoles) de Naples (1224) et dans le monopole qu'il recevait, hors médecine, sur l'enseignement supérieur. Par-delà, Frédéric II prenait une place majeure dans la mise en pratique de la renaissance juridique. Conjointement, il franchissait une étape notable dans la mobilisation par les pouvoirs de légistes et autres lettrés.

Ici réside une thèse essentielle de F. Delle Donne, exprimée par le titre de son livre : «La porte du savoir ». Frédéric II entreprit de substituer, pour son service, les grands féodaux par des hommes instruits, de plus modeste extraction, en faisant de la science un facteur de noblesse. L'on pourrait éventuellement signaler davantage que cette promotion des lettrés parachevait un large mouvement. La croyance que la vraie noblesse venait de la vertu était un poncif depuis l'Antiquité (Marvin N. Colker, " De Nobilitate Animi », Mediaeval Studies, 23, 1961, p. 47-79), et la conviction d'une noblesse du droit s'esquissait dès la fin $\mathrm{du} \mathrm{XII}^{\mathrm{e}} \mathrm{s}$. (Patrick Gilli, La noblesse $d u$ droit: débats et controverses sur la culture juridique et le rôle des juristes dans l'Italie médiévale [xii ${ }^{e}$ $X V^{e}$ siècles], Paris, Honoré Champion [Études d'histoire médiévale, 7], 2003, p. 72-76). Chez Frédéric II, cependant, la redéfinition de la noblesse s'inscrivait dans l'immense espoir d'un contrôle plénier du temporel.

Il s'articulait avec les « curiosités scientifiques de l'empereur », qui allaient de son traité Sur l'art de chasser avec des oiseaux aux traductions de textes venus du monde arabo-musulman et à une ouverture à la renaissance aristotélicienne. Le souci de maîtriser la nature, au sens médiéval, découlait de la certitude d'avoir reçu directement de Dieu l'autorité sur le monde terrestre, au point de se réclamer de l'image $\mathrm{du}$ Christ. Décortiquant proprement cette prétention à régir le temporel, F. Delle Donne en énumère encore les manifestations concrètes. Il réserve par exemple un développement approfondi au réseau de résidences et de forteresses établi dans le Mezzogiorno continental. Il en montre la fonction dans le contrôle du territoire, 
contre les interprétations ésotériques imaginées pour certains de ces édifices comme Castel del Monte. Il oppose ces constructions à l'intérêt médiocre de l'empereur pour les églises. Son soin du temporel conduit, finalement, à s'interroger sur ses sentiments religieux. Sa conduite avec juifs et musulmans en offre alors une pierre de touche.

F. Delle Donne démontre qu'il n'y eut pas tolérance, au sens actuel, mais alliage de discrimination, de raison et de pragmatisme. Rien n'est au reste plus significatif que le destin des musulmans de Sicile, déportés à Lucera, au cœur des Pouilles, par Frédéric II. Quoique leur bourreau, il s'appuya sur leur fidélité absolue, seule issue qui leur restât. Il vaudrait d'ajouter que le traité conclu par le même monarque avec le sultan al-Kâmil (1229), après une croisade « non sanglante », fut d'abord dénoncé pour scandaleux; mais Grégoire IX l'approuva quand il se réconcilia avec l'empereur. Les Latins avaient pris l'habitude de négocier avec leurs adversaires, d'après leurs intérêts (Jean Richard, Histoire des croisades, Paris, Fayard, 1996, p. 323-330).

Tout considéré, estime F. Delle Donne, Frédéric II alimenta par sa conduite les accusations portées contre lui, mais comme autant de fantasmes. Outre une certaine proximité avec l'espace musulman, dictée en partie par la géographie, ses investigations réelles ou supposées dans les mystères de la nature semblaient dénoncer son incrédulité. Qu'il se réclamât du Christ facilitait le travestissement en Antéchrist. Notons que, pourtant, la « monarchie centrée sur le Christ » était un modèle solidement implanté en Occident (Ernst Kantorowicz, The King's Two Bodies:
A Study in Mediaeval Political Theology, Princeton, University Press, 1957; reprint : 2016, p. 42-86).

Selon F. Delle Donne, Frédéric II fut, en réalité, le dernier empereur médiéval authentique par les ambitions et par les efforts. Sa revendication d'une plénitude du temporel portait quelque trace, au vrai, des précédents carolingiens et ottoniens. Et, en grand empereur chrétien, il justifiait son autorité ici-bas comme remède à la perversion de la nature humaine par le péché originel, suivant une optique augustinienne. Au reste, sa cour itinérante n'aurait su forger une « révolution culturelle», aussi réceptive qu'elle se montrât aux innovations. Pour autant, par ses succès et par son image, l'empereur devint un modèle, surtout néanmoins à travers la diffusion des lettres dues ou attribuées à Pierre des Vignes.

En bref, F. Delle Donne offre une radioscopie irréprochable de l'idéologie de Frédéric II. Elle rend caduques, en ce domaine, les simplifications sur le personnage et son milieu. Déjà indispensable à ce titre, elle laisse présager une synthèse sur le règne de l'empereur, qui achèvera de le rendre à l'histoire. Pour l'heure, elle est également une contribution importante à la culture politique sur la fin du Moyen Âge. Nous informons le lecteur que depuis la rédaction de ce compte rendu une traduction française a paru : Fulvio Delle Donne, La Porte du savoir : cultures à la cour de Frédéric II Hoenstaufen, Mich èle GrÉvin (trad.), Grenoble, UGA Éditions (Italie plurielle), 2021.

Jean-Paul Boyer

UMR 7303 - TELEMMe/Université d'Aix-Marseille 